\title{
Study of a MEMS-based Shack-Hartmann wavefront sensor with adjustable pupil sampling for astronomical adaptive optics
}

\author{
Christoph Baranec* and Richard Dekany \\ Caltech Optical Observatories, California Institute of Technology, 1200 E. California Blvd., Pasadena, California 91125, USA \\ *Corresponding author: baranec@ astro.caltech.edu
}

Received 30 July 2008; accepted 2 September 2008;

posted 5 September 2008 (Doc. ID 99583); published 25 September 2008

\begin{abstract}
We introduce a Shack-Hartmann wavefront sensor for adaptive optics that enables dynamic control of the spatial sampling of an incoming wavefront using a segmented mirror microelectrical mechanical systems (MEMS) device. Unlike a conventional lenslet array, subapertures are defined by either segments or groups of segments of a mirror array, with the ability to change spatial pupil sampling arbitrarily by redefining the segment grouping. Control over the spatial sampling of the wavefront allows for the minimization of wavefront reconstruction error for different intensities of guide source and different atmospheric conditions, which in turn maximizes an adaptive optics system's delivered Strehl ratio. Requirements for the MEMS devices needed in this Shack-Hartmann wavefront sensor are also presented. (C) 2008 Optical Society of America
\end{abstract}

OCIS codes: $\quad 010.1080,010.7350,220.2740$.

\section{Introduction}

The majority of astronomical adaptive optics systems use Shack-Hartmann sensors for wavefront measurement. In a traditional Shack-Hartmann system, an array of lenses subdivides the pupil of the optical system, each lens forming an individual image of the source. The relative location of the images can be used to determine the gradient of the local wavefront. In a more recent version of a Shack-Hartmann sensor, a prism array is placed at a pupil to impart different local tilts to the incoming wavefront, with a downstream focusing element creating an array of images [1]. In both types of Shack-Hartmann sensors, the pupil is subdivided by a static refractive optical element; however, this can also be accomplished with a segmented mirror array. An example of this is the reflective Shack-Hartmann wavefront sensor first used at the six $1.83 \mathrm{~m}$ mirror Multiple Mirror Telescope (MMT) in 1994 with a modified Schwarzschild system [2].

0003-6935/08/285155-08\$15.00/0

(C) 2008 Optical Society of America
Astronomical adaptive optics systems operate routinely in a photon-starved regime, with either natural or laser guide sources. This necessitates using the available information in an optimum way. The error associated with the wavefront sensor subsystem comprises errors dependent on the both the frame rate and the number of spatial samples across the pupil. Observations made with guide sources of varying brightness and angular extent, as well as under different seeing conditions, can be optimized to give the minimum possible residual error with control over each of these factors.

\section{Optimization of Spatial and Temporal Pupil Samplings}

An adaptive optics system's delivered science Strehl ratio is determined by the sum of all residual error sources produced by the adaptive optics system, telescope, science instrument, and atmosphere. Minimizing the error in measurement of the guide star's wavefront, the wavefront reconstruction error-while holding the other errors constantcan lead to increased adaptive optics system performance and higher Strehl ratios. Wavefront 
reconstruction error, $\sigma_{\mathrm{wf}}$, for a Shack-Hartmann wavefront sensor, is the quadratic sum of fitting error $\left(\sigma_{\mathrm{fit}}\right)$, measurement error $\left(\sigma_{\mathrm{me}}\right)$, and time-delay error $\left(\sigma_{\mathrm{td}}\right):$

$$
\sigma_{\mathrm{wf}}^{2}=\sigma_{\mathrm{fit}}^{2}+\sigma_{\mathrm{me}}^{2}+\sigma_{\mathrm{td}}^{2} .
$$

Here we examine wavefront reconstruction error in terms of spatial and temporal pupil sampling and show how each sampling term can be optimized to minimize the error in the adaptive optics system. Hardy [3] gave the following expressions for fitting error and measurement error (Eqs. 6.36, 5.13, 9.58):

$$
\begin{gathered}
\sigma_{\text {fit }}^{2}=a_{F}\left(d / r_{0}\right)^{5 / 3}, \\
\sigma_{\mathrm{me}}^{2}=\left\{\begin{array}{cc}
\left(\frac{\pi^{2}}{4 \mathrm{SNR}}\right)^{2}\left(\left(\frac{3 d}{2 r_{0}}\right)^{2}+\left(\frac{\theta d}{\lambda}\right)^{2}\right) E, & \text { for } r_{0}<d \\
\left(\frac{\pi^{2}}{4 \mathrm{SNR}}\right)^{2}\left(\left(\frac{3}{2}\right)^{2}+\left(\frac{\theta d}{\lambda}\right)^{2}\right) E, & \text { for } d<r_{0}
\end{array}\right.
\end{gathered}
$$

where $a_{F}$ is the wavefront sensor fitting error coefficient, $r_{0}$ is Fried's parameter, $d$ is the size of a subaperture at the pupil, $\theta$ is the angular size of the guide source, $d_{\text {tele }}$ is the diameter of the telescope, and $E$ is the error propagator. The signal-to-noise ratio (SNR) of a single subaperture slope measurement in the absence of background is simplified to ([3], Eq. 5.17),

$$
\mathrm{SNR}=\frac{n_{\mathrm{phot} / \mathrm{sub}}}{\left(n_{\mathrm{phot} / \mathrm{sub}}+n_{\mathrm{pix}}^{2} e^{2}\right)^{1 / 2}},
$$

where $n_{\text {phot/sub }}$ is the number of detected photoelectrons per subaperture, $n_{\text {pix }}$ is the number of detector pixels across a subaperture, and $e$ is the read noise in electrons per pixel.

Time-delay error can be expressed as ([3] Eq. 9.56),

$$
\sigma_{\mathrm{td}}^{2}=\left(\tau_{s} / \tau_{0}\right)^{5 / 3},
$$

where $\tau_{0}$ is a time constant and $\tau_{s}$ is the time delay between wavefront measurement and correction in the adaptive optics control system. The time delay can be expressed as the number of frames of delay in the adaptive optics control loop, $n_{f \text { delay }}$, divided by the frame rate, $f$, because the time delay is typically limited by integration and readout of the wavefront sensor detector.

Spatial pupil sampling $(s)$ of the wavefront by a Shack-Hartmann sensor is equal to the ratio of telescope and subaperture diameters, as $s=d_{\text {tele }} / d$, with the number of detected photoelectrons per subaperture given by

$$
n_{\mathrm{phot} / \mathrm{sub}}=\frac{4}{\pi} \frac{\Phi}{s^{2} f}
$$

where $\Phi$ is the product of the incident radiant flux of photons and telescope adaptive optics system and detector quantum efficiencies.

The values of $s$ and $f$ that minimize $\sigma_{\mathrm{wf}}^{2}, s_{\text {opt }}$, and $f_{\text {opt }}$, respectively, can be evaluated after substituting $s$ into Eqs. (2) and (3). For the case of $d<r_{0}$ (necessary for high-contrast imaging and precision astrometry [4-8]) the analytic expressions for $s_{\text {opt }}$ and $f_{\text {opt }}$ are

$$
\begin{gathered}
s_{\text {opt }}=0.529 \frac{a_{F}^{12 / 35} d_{\text {tele }}^{4 / 7} \tau_{0}^{3 / 14} \Phi^{3 / 14}}{r_{0}^{4 / 7} n_{\text {fdelay }}^{3 / 14}} \\
f_{\text {opt }}=0.801 \frac{n_{\text {fdelay }}^{1 / 14} r_{0}^{3 / 7} \Phi^{3 / 14}}{a_{F}^{9 / 35} d_{\text {tele }}^{3 / 7} \tau_{0}^{11 / 14}}
\end{gathered}
$$

where $s_{\text {opt }}$ and $f_{\text {opt }}$ are found by solving the system of equations

$$
\frac{d}{d s}\left[\sigma_{w f}^{2}\right]=0, \quad \frac{d}{d f}\left[\sigma_{w f}^{2}\right]=0
$$

by the least-squares method and assuming no noise with $\theta=0$ and $E \sim 1$. (The error propagator can be expressed as $E=\kappa_{1}+\kappa_{2} \ln (s)$. Based on a fit of values presented in [3] , it is found that $\kappa_{1}=0.19$ and $\kappa_{2}=0.21$. Note that the error propagator varies slowly from 0.5 to 1.1 for values of $s$ from 5 to 64 .)

These closed-form equations are functionally equivalent to those calculated by Angel [8] for optimal spatial and temporal sampling with a Mach-Zehnder wavefront sensor, although $\tau_{0}$ and loop delay are explicitly called out here. Also, unlike in Angel, Eqs. (7) and (8) do not assume a frozen flow of turbulence, and therefore there is no direct relation between $\tau_{0}$ and $r_{0}$. However, no closed-form solutions for $s_{\text {opt }}$ and $f_{\text {opt }}$ exist when taking into account the error propagator and read noise. When $d>r_{0}$, which is true of most current astronomical adaptive optics systems, $s_{\text {opt }}$ and $f_{\text {opt }}$ must also be evaluated numerically.

Figure 1 shows $\sigma_{\mathrm{wf}}$ as a function of $s$-where the frame rate has been numerically reoptimized at each point-for a $10 \mathrm{~m}$ telescope for all values of $d$ and including the error propagator. Shown are curves with different magnitudes of stellar guide source, under different seeing conditions and with two different detector technologies: a standard CCD with $5 e^{-}$of read noise and an electron multiplying CCD (EMCCD) with zero read noise. The EMCCD, however, suffers from an excess noise factor, reducing the SNR by $\sqrt{2}$ as a result of its stochastic gain mechanism[9]. Table 1 describes the minimum of each curve in terms of $\sigma_{\mathrm{wf}}, s_{\mathrm{opt}}$, and $f_{\mathrm{opt}}$ for guide sources of visual magnitude 7, 11, and 15. Table 1 also shows the range of values of $s$ that correspond to no greater than a $5 \%$ error in $\sigma_{\mathrm{wf}}^{2}$. It can be seen in Fig. 1 that when guiding on bright guide sources $\left(m_{V} \sim 7\right)$, it is always best to use the highest possible pupil 

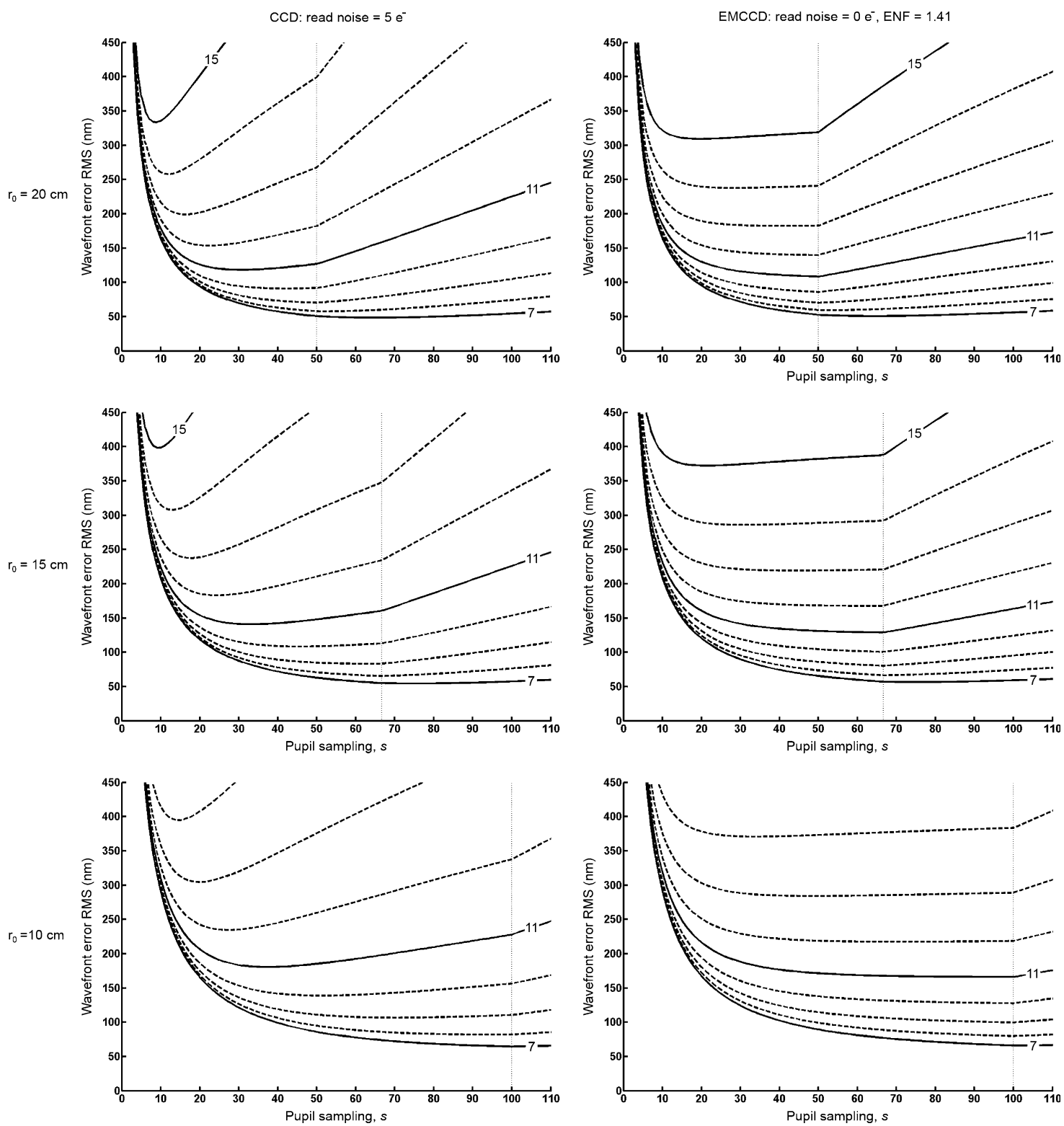

Fig. 1. Wavefront reconstruction error as a function of pupil sampling, $s$, for two different detectors, a CCD with $5 e^{-}$read noise (left) and an electron-multiplying CCD with zero read noise and an excess noise factor of $\sqrt{2}$, and during excellent $\left(r_{0}=20 \mathrm{~cm}\right.$ at $\lambda=500 \mathrm{~nm}$, top), median $\left(r_{0}=15 \mathrm{~cm}\right.$, middle) and poor $\left(r_{0}=10 \mathrm{~cm}\right.$, bottom) seeing conditions. The vertical dotted line in each plot represents $s=d_{\text {tele }} / r_{0}$. The curves indicate integer magnitudes of the guide source, with $m_{V}=7, m_{V}=11$, and $m_{V}=15$ labeled explicitly. All assume a combined telescope and adaptive optics system transmission of $22 \%$, detector quantum efficiency of $80 \%, d_{\text {tele }}=10 \mathrm{~m}, \theta=0, a_{F}=0.28, n_{\text {pix }}=2$, and $\tau_{0}=3 \mathrm{~ms}$ with a two-frame time delay.

sampling because the wavefront error is dominated primarily by fitting error. For the case of a CCD guiding on dim sources $\left(m_{V}>11\right)$, it is necessary to have fine adjustment of pupil sampling. For each brightness of guide source, there is only a narrow range of $s$ that avoids extra wavefront reconstruction error. A CCD-based Shack-Hartmann wavefront sensor would therefore benefit from the ability to have fine control over low spatial pupil sampling as well as having a high spatial pupil sampling mode for use with bright sources. An EMCCD-based wavefront sensor, however, has a shallow minimum in its wavefront error as a function of $s$ for dimmer stars. Therefore, it may be more important to choose the spatial pupil sampling of the wavefront sensor based on other system considerations.

Note that when guiding is on extended sourcesincluding laser guide stars-where $\theta>0$, the $\sigma_{\mathrm{me}}^{2}$ term is increased in the calculation of total wavefront reconstruction error. This causes the curves to shift up overall in Fig. 1; however, $s_{\text {opt }}$ and $f_{\text {opt }}$ can either increase or decrease depending on the guide source brightness.

\section{Possible Implementations of an Adjustable Pupil Sampling Shack-Hartmann Wavefront Sensor}

NAOS (the Nasmyth adaptive optics system) [10], in recognition of the advantage of a lower wavefront 
Table 1. Optimal Spatial Pupil Sampling and Frame Rates ${ }^{a}$

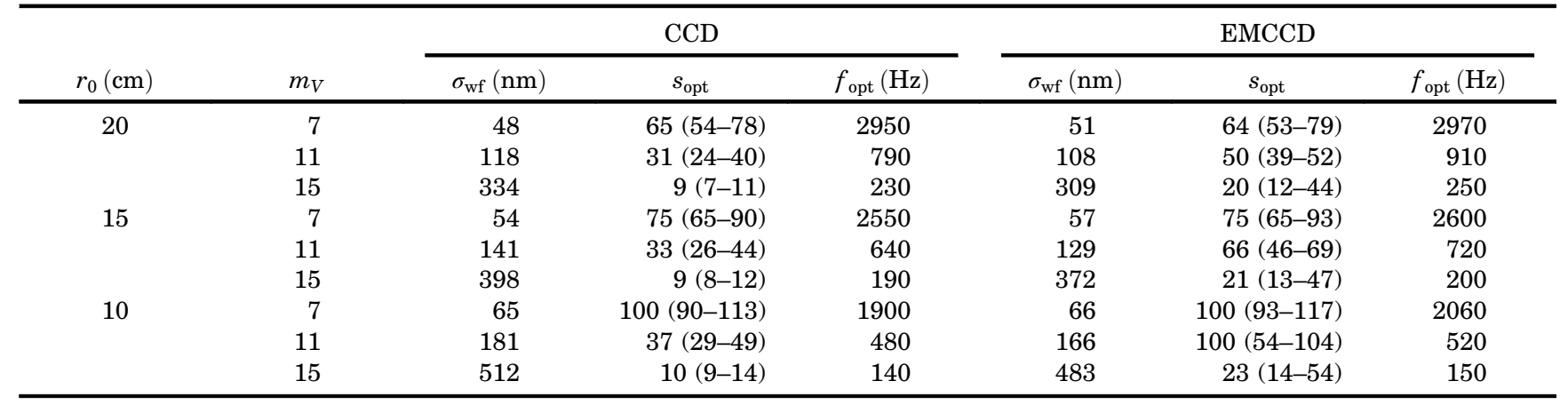

${ }^{a}$ These values describe the minima in wavefront reconstructor error curves presented in Fig. 1 . Parenthesized terms show the range of $s$ such that $\sigma_{\mathrm{wf}}^{2}$ is within $5 \%$ of its minimum value.

reconstructor error with an adjustable pupil sampling wavefront sensor, has implemented a mechanical lenslet-array exchanger that is used to change pupil sampling to either $14 \times 14$ or to $7 \times 7$, depending on observing conditions. Rousset et al. [11] described how the $14 \times 14$ sampling should result in a higher delivered Strehl ratio from the adaptive optics system when using guide stars of visual magnitude 8 to 13 , and, similarly, the $7 \times 7$ sampling should give better performance when guiding with dimmer guide stars of visual magnitude 14 to 18 . This advantage has also been identified in the future upgrades to the $5.1 \mathrm{~m}$ Hale and $10 \mathrm{~m}$ Keck adaptive optics systems $[\underline{4}, \underline{6}, \underline{7}, \underline{12}]$, which will each include four different lenslet arrays in mechanical lenslet-array exchangers, enabling pupil spatial sampling options from $8 \times 8$ up to $64 \times 64$. Drawbacks to this approach include tight tolerances and additional degrees of freedom needed for both exchanging and aligning the lenslet arrays within the wavefront sensor, as well manufacturing costs for custom lenslet arrays, one for each pupil sampling desired.

Rha et al. [13] have demonstrated the technique of adjustable pupil sampling using a liquid crystal device (LCD) to create a closed-loop adaptive optics system capable of changing from a $2 \times 2$ to a $4 \times 4$ Shack-Hartmann wavefront sensor. Unfortunately, the technique has not garnered much use in the astronomical community, mainly because LCDs have many limitations for astronomy. These include narrow spectral and polarization bandwidth and the limitation of introducing only $2 \pi$ phase shifts that can lead to undesirable diffraction effects. Zhao et al. [14] found that in order to achieve 95\% diffraction efficiency with an LCD spatial light modulator used as a lenslet array, at least eight phase levels are needed per Fresnel zone, leading to a requirement for a large number of LCD pixels for each subaperture. Furthermore, it was found that the LCD's diffraction inefficiencies caused stray spots to land on the detector, which increased noise in a poorly understood way on the subaperture slope measurements. The LCD pixel-number requirements and diffraction effects would also be present if the LCD were used to emulate a prism array instead of a lenslet array.

We propose the use of MEMS segmented mirror arrays for pupil sampling adjustment in a Shack-Hartmann wavefront sensor. A conceptual design for a Shack-Hartmann wavefront sensor with a segmented mirror array is presented in Fig. 2 . The wavefront sensor is a drop-in replacement for conventional wavefront sensors, being optically downstream of an adaptive optics system's deformable mirror and beam splitter. The collimating lens images the pupil onto the segmented mirror array. Tilt is then added to each subaperture by tilting the individual or groups of segments, and an imaging lens focuses the light at the image plane. An optional relay system can be used to demagnify the Shack-Hartmann pattern before it is imaged onto the detector.

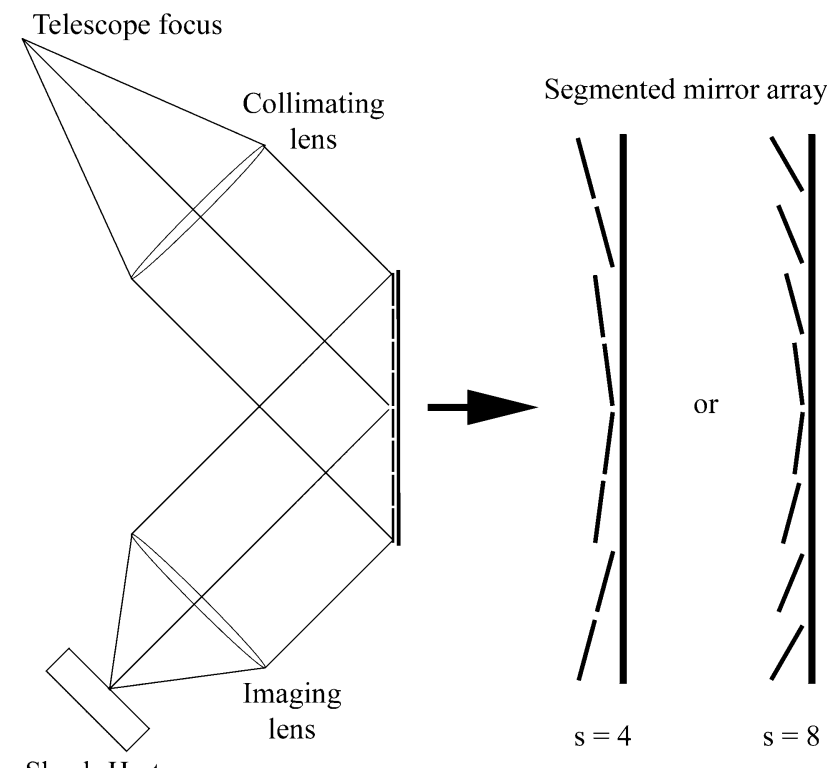

Shack-Hartmann

pattern on detector

Fig. 2. Conceptual design of a Shack-Hartmann wavefront sensor using a segmented mirror array to adjust spatial pupil sampling. The array is shown with piston control of the segments. Angles are exaggerated, not to scale. 
Although others have suggested MEMS for use in Shack-Hartmann wavefront sensors for other purposes $[15,16]$, none have previously considered the benefits of MEMS for minimizing wavefront reconstruction error. Andrews et al. [17] conducted experiments using a MEMS device in a Shack-Hartmann wavefront sensor; however, they did not consider the advantages of pupil reconfiguration. MEMS segmented mirror arrays offer a high-throughput, achromatic, and polarization- and wavelengthindependent solution for adjustable pupil subdivision without the need of an exchange mechanism. Using MEMS, a Shack-Hartmann wavefront sensor with redefinable pupil sampling, as envisioned by Rha et al., can be implemented in an astronomical adaptive optics system.

Not only can pupil sampling be adjusted to optimize the adaptive optics system's Strehl ratio when changing to different magnitudes of natural guide star, it can also be used to adapt to dynamic flux conditions. As an example, sodium $D_{2}(589 \mathrm{~nm})$ laser guide beacons can fluctuate in brightness by a factor of two over several minutes $[18,19]$. The pupil sampling could be adjusted in real-time with the MEMS device (quickly switching $s$ between frame integrations) to adapt to the changing return flux levels. MEMS-based wavefront sensors have additional advantages, including non-common-path calibration, increased slope linearity, reduced detector format size, complete pupil sampling, and pulse tracking of laser guide beacons; each of these potentially improve adaptive optics system performance compared with fixed-geometry lenslet- or prismbased Shack-Hartmann wavefront sensors.

Non-common-path errors between the science instrument and wavefront sensor can be changed by adjusting the tilts of individual subapertures $[2,16]$. By using an internal calibration source at a focal plane, the slope of each subaperture can be measured by calculating the subimage's position. Each subimage can be steered to a new position by the MEMS segmented mirror array, effectively adding an offset that can be used either to eliminate non-common-path errors or to add optical path differences between the wavefront sensor and science instrument for other purposes $[20,21]$.

An advantage of canceling out the non-commonpath errors in this way is that each ShackHartmann image can be shifted to operate on pixel intersections where the transfer curves for quad cells are in a linear regime [3]. This can be achieved by running the adaptive optics system in a closed loop on an internal calibration source and adding slope offsets to the wavefront sensor until the image on the science detector is corrected. The deformable mirror position is then measured over the length of time required to reduce noise effects. The loop is then opened, and the deformable mirror is set to the measured mean position. The individual subapertures of the MEMS array are then driven in tip and tilt such that their corresponding images shift to pixel bound- ary intersections on the detector. This process must be repeated if the transfer curve nonlinearities have affected the slope offsets until the system can be run in closed loop by simply driving the ShackHartmann subimages to pixel intersections. Operating the adaptive optics system on-sky in this way simplifies the control law and real-time computational requirements by obviating the need for a nonlinear correction term in the slope calculation.

The ability to reposition Shack-Hartmann images on the detector by adding tilts to each subaperture can also reduce the required detector format size. In a typical Shack-Hartmann wavefront sensor, a circular pupil is imaged onto a square detector; thus the corners of the detector are not used. By intelligently repositioning the Shack-Hartmann images such that the final subimage pattern is square, the requirement for the areal format of the detector can be reduced by up to $(1-\pi / 4)=21 \%$. Alternatively, for a given commercially available wavefront sensor detector, more physical subapertures could be sensed. As an example, an $80 \times 80$ format CCD with $4 \times 4$ pixel subapertures would be limited to $s=20$, with only 316 of the 400 potential subapertures illuminated. By using a MEMS segmented mirror array to reposition the subimages, sampling of $s=22$ (totaling 380 illuminated subapertures) could be achieved with the same format detector.

Square geometry Shack-Hartmann wavefront sensors typically suffer from information loss around the edge of the pupil as a result of the projection of an annular pupil onto a square grid of subapertures. Measurements from the edge subapertures are generally disregarded because the SNRs are much lower than those of fully illuminated subapertures. This loss of information results in additional error in the wavefront estimate because the reconstructor must extrapolate into these unsensed areas of the pupil. A segmented mirror array can be used to define custom edge subapertures such that the light of partially illuminated segments is combined with that of neighboring segments, increasing the subaperture's SNR. Figure 3 shows an example of traditional edge subapertures with light loss, and combined-segment subapertures that capture all of the light. The custom-defined edge subapertures allow for complete wavefront measurement and better edge control of the adaptive optics system's deformable mirror.

The tip and tilt control over each subaperture can also be used to compensate for perspective elongation when using pulsed-laser guide beacons [15, 16$]$. By dynamically driving each subaperture with a predetermined open-loop signal that-in the absence of atmospheric wavefront aberration-keeps the corresponding beacon image centered on the subaperture's optical axis, the effects of perspective elongation can be mitigated. 

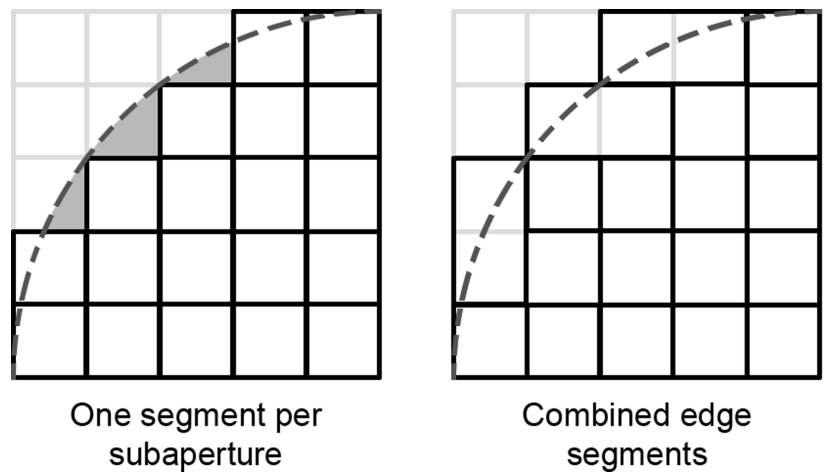

Fig. 3. Configuration of subapertures (left) for a traditional Shack-Hartmann wavefront sensor and (right) for a MEMS segmented mirror array with combined edge segments. The footprint of the pupil is outlined in dashed gray, and subapertures are outlined in black. The light lost in a traditional Shack-Hartmann wavefront sensor is seen as the gray area.

\section{Design of a MEMS-Based Shack-Hartmann Wavefront Sensor}

The first-order optical design for a Shack-Hartmann wavefront sensor using a lenslet array is highly constrained, given the telescope diameter, focal length, detector dimensions, desired plate scale, and desired pupil sampling. This is similarly true for a sensor using a segmented mirror array. The equations for the focal length of the collimating and imaging lens, $f_{\text {col }}$ and $f_{\text {img }}$, respectively, and the minimum surface tilt angle required on the edge subaperture to adequately separate the Shack-Hartmann spot pattern on the detector, $\phi$, are

$$
\begin{gathered}
f_{\text {col }}=\frac{s d_{\text {sm }} f_{\text {tele }}}{d_{\text {tele }}}, \\
f_{\text {img }}=\frac{s d_{\mathrm{sm}} p}{d_{\text {tele }} \alpha m}, \\
\phi=\frac{(s-1)}{s} \frac{\left(n_{\text {pix }} \alpha\right)}{4} \frac{d_{\text {tele }}}{d_{\text {sm }}},
\end{gathered}
$$

Table 2. Stroke Requirements for a Segmented Deformable Mirror ${ }^{a}$

\begin{tabular}{rccccc}
\hline$s$ & Telescope & $d_{\text {tele }}(\mathrm{m})$ & $\alpha(\operatorname{arcsec} /$ pixel $)$ & $n_{\text {pix }}$ & Stroke $(\mu \mathrm{m})$ \\
\hline 9 & SOAR & 4.1 & 0.4 & 6 & 10.6 \\
9 & Hale & 5.1 & 1.3 & 2 & 14.3 \\
9 & MMT & 6.5 & 0.4 & 6 & 16.8 \\
$\mathbf{6 4}$ & Hale & 5.1 & $2.1^{b}$ & 2 & 25.6 \\
$\mathbf{6 4}$ & Keck & $11^{c}$ & 1.0 & 2 & 26.1 \\
$\mathbf{6 4}$ & TMT & 30 & 0.5 & 4 & $\mathbf{7 1 . 6}$ \\
\hline
\end{tabular}

${ }^{a}$ Values in bold exceed current technology capability.

${ }^{b}$ This plate scale has been matched to the diffraction limited point spread function size.

${ }^{c}$ Keck's maximum diameter. where $d_{\mathrm{sm}}$ is the diameter of each mirror segment of the mirror array, $f_{\text {tele }}$ is the telescope focal length, $p$ is the pixel size, $\alpha$ is the plate scale in radians per pixel, and $m$ is the magnification of the relay. The focal length of the imaging lens is arbitrarily adjustable, as it is dependent on the magnification of the relay, a free parameter. The minimum angle of tilt required on the edge subaperture, however, is highly constrained by the telescope diameter. Given a desired pupil sampling and Shack-Hartmann image spacing, $n_{\text {pix }} \alpha$, the required amount of tilt is proportional to the ratio of the telescope diameter to the segment diameter. From Eq. (11), the product $\phi d_{\mathrm{sm}}$, which for a mirror tilted about one edge is equal to the actuator stroke, is seen to be proportional to the telescope diameter. Requirements for tilt and actuator stroke for segmented mirror arrays are presented for a few example applications in Table 2 . These stroke requirements could be relaxed if the actuators could be made to cantilever each mirror segment, but this may introduce manufacturing complications.

If the segments can physically piston, e.g., if they are mounted on three independent actuators, grouped subapertures can be phased, creating continuous surfaces. This is achieved without increasing the stroke requirement on the device. When segments are grouped together to form larger effective subapertures-while holding $\alpha$ and $n_{\text {pix }}$ constantthe effective $d_{\mathrm{sm}}$ increases, and $\phi$ decreases inversely, with the overall stroke requirement decreasing only slightly as $(s-1) / s$. This can be seen graphically in Fig. 2, wherein the maximum stroke required is approximately equal for both the $s=4$ and $s=8$ modes of the mirror array; the segment tilts of the $s=4$ mode are the same as the tilts on the centermost four segments in the $s=8$ mode.

Special consideration must be taken with a segmented mirror array with only tip-tilt actuation when combining segments into subaperture groups. A relative phase delay will be introduced between segments of a subaperture, causing extra structure to be created in each subaperture's point spread function, decreasing the SNR in the subsequent subaperture slope measurement. When guiding on coherent sources, such as natural guide stars, this is of concern when the full width at half-maximum of the diffraction-limited point spread function created by a single segment is greater than the atmospheric seeing width. However, this effect is lessened when guiding on laser guide stars or solar system objects with large angular extents because of their spatial incoherence. Alternatively, a tip-tilt segmented mirror array, designed such that individual square mirror segments in groups of $2 \times 2$ all have their fulcrum at the middle intersection of the group, could be used to switch spatial pupil sampling by a factor of two while still maintaining phase coherency across a grouped subaperture.

A tip-tilt-piston MEMS device that is appropriate for astronomical adaptive optics has been fabricated by Sandia National Laboratories [22]. The mirror 
has the following characteristics: $d_{\mathrm{sm}}=0.5 \mathrm{~mm}$, $s=9$, a maximum stroke of $26.7 \mu \mathrm{m}$, an allowable tilt of $\sim 3^{\circ}$, and a control rate of at least $1 \mathrm{kHz}$. While the spatial sampling of this device is smaller than that of many astronomical adaptive optics systems, it can be used without modification at moderately sized telescopes such as the $4.1 \mathrm{~m}$ Southern Astrophysical Research (SOAR), $5.1 \mathrm{~m}$ Hale, and current $6.5 \mathrm{~m}$ MMT. Simply increasing the number of segments in a device can meet the requirements for a wavefront sensor with 64 subapertures across the pupil at both the Hale[4,12] and the Keck[6,7] telescopes. The use of a segmented mirror array wavefront sensor for the next generation of extremely large telescopes (e.g., the Thirty Meter Telescope (TMT) [23]), however, requires a significant breakthrough in actuator technology, because the requirements for actuator stroke are almost a factor of 3 greater.

\section{Conclusion}

It has been shown that a Shack-Hartmann sensor using a MEMS segmented mirror array in place of the traditional lenslet array has the potential to optimize spatial sampling at the pupil for any given brightness of guide source and observing condition. This serves to minimize total wavefront reconstruction error, which in turn maximizes an adaptive optics system's delivered Strehl ratio. Such a sensor also has the ability to calibrate non-common-path errors with additional static tilts on individual segments that can be used to linearize the subimage slope measurements. The segment tip-tilt control can be used to reshape the Shack-Hartmann pattern so that it uses more area of the detector. A MEMS segmented mirror array can also be used combine edge segments into subapertures, fully sampling the pupil with no information loss around edges. Additionally, the arrays can be used to dynamically correct for perspective elongation of pulsed laser beacons.

Recent innovation has made it possible to field the required devices in MEMS-based Shack-Hartmann wavefront sensors on moderately sized telescopes optimized for faint guide stars. An increase in the element number of these devices will lead to improved adaptive optics performance and additional engineering benefits to all current telescope apertures and all guide star brightnesses. Additional stroke capability will also extend these gains and benefits to the next generation of extremely large telescopes.

This work has been supported by the National Science Foundation (NSF) under grant AST0619922.

\section{References}

1. N. Putnam, M. Snyder, T. Stalcup, and R. Angel, "Manufacture and use of a Shack-Hartmann sensor with a multifaceted prism for simultaneous sensing of multiple wavefronts," Proc. SPIE 5490, 1138-1145 (2004).

2. D. Colucci, M. Lloyd-Hart, D. Wittman, R. Angel, A. Ghez, and B. McLeod, "A reflective Shack-Hartmann wave-front sensor for adaptive optics," Publ. Astron. Soc. Pac. 106, 11041110 (1994).

3. J. W. Hardy, Adaptive Optics for Astronomical Telescopes (Oxford U. Press, 1998).

4. R. Dekany, A. Bouchez, M. Britton, V. Velur, M. Troy, J. C. Shelton, and J. Roberts, "PALM-3000: visible-light AO on the 5.1 Meter Telescope," Proc. SPIE 6272, 62720G (2006).

5. R. Dekany, "Exoearth study with TMT," Proc. SPIE 5382, 12-20 (2004).

6. P. Wizinowich, R. Dekany, D. Gavel, C. Max, S. Adkins, B. Bauman, J. Bell, A. Bouchez, M. Britton, J. Chin, R. Flicker E. Johansson, R. Kupke, D. Le Mignant, C. Lockwood, D. Medeiros, E. McGrath, A. Moore, C. Neyman, M. Reinig, and V. Velur, "W. M. Keck Observatorys next-generation adaptive optics facility," Proc. SPIE 7015, 701511 (2008).

7. D. Gavel, R. Dekany, C. Max, P. Wizinowich, S. Adkins, B. Bauman, J. Bell, E. Johansson, R. Kupke, C. Lockwood, A. Moore, C. Neyman, M. Reinig, and V. Velur, "Concept for the Keck next-generation adaptive optics system," Proc. SPIE 7015, 701567 (2008).

8. J. R. P. Angel, "Ground-based imaging of extrasolar planets using adaptive optics," Nature 368, 203-207 (1994).

9. A. G. Basden, C. A. Haniff, and C. D. Mackay, "Photon counting strategies with low-light-level CCDs," Mon. Not. R. Astron. Soc. 345, 985-991 (2003).

10. P. Feautrier, P. Kern, R. Dorn, G. Rousset, P. Rabou, S. Laurent, J.-L. Lizon, E. Stadler, Y. Magnard, O. Rondeaux, M. Cochard, D. Rabaud, A. Delboulbe, P. Puget, and N. Hubin, "The NAOS visible wavefront sensor," Proc. SPIE 4007, 396407 (2000).

11. G. Rousset, F. Lacombe, P. Puget, E. Gendron, R. Arsenault, P. Kern, D. Rabud, P.-Y. Madec, N. Hubin, G. Zins, E. Stadler, J. Charton, P. Gigan, P. Feautrier, "Status of the VLT Nasmyth Adaptive Optics System (NAOS)," Proc. SPIE 4007, 72-81 (2000).

12. C. Baranec, "High-order wavefront sensing system for PALM3000," Proc. SPIE 7015, 70155M (2008).

13. J. Rha, D. Volez, and M. Giles, "Reconfigurable ShackHartmann wavefront sensor," Opt. Eng. 43, 251-256 (2004).

14. L. Zhao, N. Bai, X. Li, L. Ong, Z. Fang, and A. Asundi, "Efficient implementation of a spatial light modulator as a diffractive optical microlens array in a digital Shack-Hartmann wavefront sensor,” Appl. Opt. 45, 90-94 (2006).

15. C. Baranec, B. J. Bauman, and M. Lloyd Hart, "Concept for a laser guide beacon Shack-Hartmann wave-front sensor with dynamically steered subapertures," Opt. Lett. 30, 693-695 (2005).

16. B. J. Bauman, Optical design for Extremely Large Telescope adaptive optics systems," Ph.D. dissertation (University of Arizona, 2003).

17. J. Andrews, S. W. Teare, S. R. Restiano, T. Martinez, C. Wilcox, and D. Wick, "A high speed reflective wave front sensor using a novel MEM device," in 2007 IEEE Aerospace Conference (IEEE, 2007), pp. 1-6.

18. C. O'Sullivan, R. M. Redfern, N. Ageorges, H.-C. Holstenberg, W. Hackenberg, T. Ott, S. Rabien, R. Davies, and A. Eckart, "Short timescale variability of the mesospheric sodium layer," Exp. Astron. 10, 147-156 (2000).

19. L. C. Roberts and L. W. Bradford, "Measurements of mesospheric sodium abundance above the Hawaiian Islands," Publ. Astron. Soc. Pac. 119, 787-792 (2007).

20. M. Kenworthy, P. Hinz, R. Angel, A. Heinze, and S. Sivanandam, "Whack-a-speckle: focal plane wavefront sensing in theory and practice with a deformable secondary mirror and 5 micron camera," Proc. SPIE 6272, 62723K (2006).

21. E. Serabyn, K. Wallace, M. Troy, B. Mennesson, P. Haguenauer, R. Gappinger, and R. Burruss, "Extreme 
adaptive optics imaging with a clear and well-corrected offaxis telescope subaperture," Astrophys. J. 658, 1386-1391 (2007).

22. D. Dagel, W. Cowan, O. Spahn, G. Grossetete, A. Griñe, M. Shaw, P. Resnick, and B. Jokiel, "Large-stroke mems deformable mirrors for adaptive optics," J. Microelectomech. Syst. 15, 572-583 (2006).

23. B. L. Ellerbroek, C. Boyer, C. Bradley, M. C. Britton, S. Browne, R. A. Buchroeder, J.-L. Carel, M. K. Cho,
M. R. Chun, R. Clare, R. Conan, L. G. Daggert, R. G. Dekany, J. H. Elias, D. A. Erickson, R. Flicker, D. T. Gavel, L. Gilles, P. Hampton, G. Herriot, M. R. Hunten, R. R. Joyce, M. Liang, B. A. Macintosh, R. Palomo, I. P. Powell, S. C. Roberts, E. Ruch, J.-C. Sinquin, M. J. Smith, J. A. Stoesz, M. Troy, G. A. Tyler, J.-P. Veran, C. R. Vogel, and Q. Yang, "A conceptual design for the Thirty Meter Telescope adaptive optics systems," Proc. SPIE 6272, 62672I (2006). 